Address for correspondence:

Dr Hannah Wright, University of Lincoln, School of Life Sciences, Joseph Banks Laboratories, LN6 7DL, UK E-mail: hwright@lincoln.ac.uk Richard Mills was affiliated to the National Autistic Society, London, UK, during the time of this study.

\section{Pet Dogs Improve Family Functioning and Reduce Anxiety in Children with Autism Spectrum Disorder}

\author{
Hannah Wright", Sophie Hall", Annette Hames*, \\ Jessica Hardiman ${ }^{*}$, Richard Mills ${ }^{\ddagger}$, PAWS Project \\ Team $^{\dagger}$ and Daniel Mills ${ }^{*}$ \\ "University of Lincoln, School of Life Sciences, Joseph Banks \\ Laboratories, UK \\ ${ }^{\dagger}$ Dogs for the Disables, Frances Hay Centre, Banbury, Oxfordshire, UK \\ ${ }^{\ddagger}$ Research Autism, Adam House, London, UK
}

ABSTRACT There is increasing evidence to suggest that dogs are beneficial for children with Autism Spectrum Disorder (ASD) in therapy sessions, and anecdotal reports suggest that dogs may have wider benefits, in a family setting. This study investigated the effect of dog ownership on family functioning and child anxiety. Using a validated scale of family strengths and weaknesses (Brief Version of the Family Assessment Measure-III [General Scale]), we compared parents of children with ASD who had recently acquired a pet dog ( $n=42$, Intervention group) with a similar group of parents not acquiring a dog ( $n=28$, Control group) at matched time points. A sub-population ( $n=14$ acquiring a dog, $n=26$ controls) completed a parental-report measure of child anxiety (Spence Children's Anxiety Scale). The primary carer completed the scales via telephone at Baseline (up to 17 weeks before acquiring a dog), Postintervention (3-10 weeks after acquisition), and Follow-up (25-40 weeks after acquisition). Over time, scores for family functioning showed significant improvements (reduced family weaknesses, increased strengths) in the dog-owning compared with the non-dog owning group. In comparison with the non-dog owning group, anxiety scores in the dog-owning group reduced by a greater percentage, most notably in the domains of Obsessive Compulsive Disorder (26\% greater decrease), Panic Attack and Agoraphobia (24\%), Social Phobia (22\%), and Separation Anxiety (22\%). The results illustrate the potential of pet dogs to improve whole family functioning and child anxiety.

Keywords: anxiety, autism, children, family-functioning, pet dogs 
Uncorrected Proof

Pet Dogs Improve Family Functioning and Reduce Anxiety in Children with Autism Spectrum Disorder

Autism Spectrum Disorder (ASD) affects 62/10,000 individuals globally (Elsabbagh et al. 2012); lasting into adulthood it costs society billions of pounds (Knapp et al. 2007). ASD is incurable, but receiving the correct levels of support considerably enhances quality of life (Batten et al. 2006, p. 3). Despite this there is concern that many individuals with ASDs do not receive sufficient support (Batten et al. 2006, p. 3; Rosenblatt 2008, pp. 5-7). Although the use of therapy dogs in autism treatment programs is of increasing scientific interest (Silva et al. 2011; Berry et al. 2013; O'Haire 2013), to date few studies have explored how pet dogs may bring similar benefits to those affected by autism. Therefore, the aim of this research was to assess the utility of acquiring a pet dog in supporting children with autism and their family members. For the purpose of this paper, we focus specifically on the effects of dog ownership on family functioning and child anxiety.

Parenting a child with autism places high strain on the family unit, even in comparison with the parenting of children with other mental or physical disorders (Dunn et al. 2001; Tonge et al. 2006). For instance, mothers of children with autism score higher on life stresses and depression scales than parents without a child with autism (Quintero 2010). Wider negative influences are also observed in the family unit, with reduced quality of life for the parents and family members living with a child with autism (Lee et al. 2007). Although traditional focus in the management of autism is predominantly patient-orientated, there is increasing interest in the role of the family and the caregiver in improving the effectiveness of behavior management (e.g., see Brereton and Tonge 2005; Tonge et al. 2006; Green et al. 2010). Indeed, research suggests an association between maternal wellbeing and sibling adjustment (Quintero 2010) and a relationship between family functioning and behavioral problems in children with autism (Sikora et al. 2013). As such there is growing awareness that autism-treatment studies should include measures of family functioning (Fisman et al. 2000; Tunali and Power 2002); this may be particularly important when looking at home-based interventions. Given that pet dogs live in the family home, it could be argued that the acquisition of a pet dog is a home-based intervention.

There is a strong association between autism and symptoms of anxiety (Ghaziuddin 2002; White et al. 2009; Mayes et al. 2011). The nature and role of anxiety in children with ASD is complex and requires further investigations. For instance, the increased social phobia associated with teenagers with ASD may reflect the heightened social challenges they face in comparison with typically developing teenagers (Tantam 2003) and the fact that it is harder for them to adapt to new routines and meet family expectations (Khouzam, El-Gabalawi and Priest 2004), rather than being inherent to the condition. Increased anxiety in children with ASD has also recently been associated with restricted interests (a typical behavioral symptom of ASD) (Spiker et al. 2012), highlighting a potential pathway for research focusing on remediating these behavioral issues (i.e., restricted interests, repetitive behaviors). Although the purpose of the present research was not to attempt to disentangle the issues surrounding anxiety and ASD, this brief summary highlights the importance of considering child anxiety in ASD treatment programs. Combined with the existing evidence base of studies which show that trained assistance dogs can reduce anxiety in children with ASD (Smyth and Slevin 2010; Viau et al. 2010), there is a strong rationale for exploring the effects of pet dogs on child anxiety.

A large number of studies involving dogs and children with ASD have focused on outcome measures directly related to the child, and are limited to the use of dogs in therapy sessions or trained assistance dogs placed in the family home (e.g., Burrows, Adams and Spiers 2008; Solomon 2010; Viau et al. 2010). The primary focus for study has been communication and social behavior of the subject children, since these are the predominant symptoms of ASD. 
Results are generally supportive of potential benefits in these domains; however, the application of findings to pet dogs remains unknown. One of the major benefits of considering pet dogs is that they are more easily accessible than trained assistance dogs, which require substantial time and economic input.

More recently, studies have begun to focus on the potential benefits of pet dogs for the child and caregivers ( Garndgeorge et al. 2012; Carlisle 2014; Wright et al. 2015), supported by previous studies involving trained assistance dogs that suggest there may be beneficial effects on the overall welfare of the family (Burrows, Adams and Spiers 2008; Viau et al. 2010). Many of these benefits may not be related to the specific training that these assistance animals receive, but rather are incidental results of the dog being present. Indeed, Grandgeorge et al. (2012) report an increase in pro-social behaviors in children with autism upon acquisition of a pet dog. Parental reports suggest that the presence of an assistance dog in the home results in reduced child anxiety, and this is supported by decreases in cortisol awakening responses in these children following placement of the dog, which increase again following removal of the animal (Viau et al. 2010). Indeed, there is mounting evidence to highlight the potential benefits of animals in reducing anxiety in a range of mental health disorders, including schizophrenia (Lang et al. 2010) and Alzheimer's Disease (Mossello et al. 2011). Other benefits reported by parents in these studies include decreased problematic behavior of the child, increased sense of security for the parents, and increased social acknowledgement for family members. These wider positive effects on the whole family could potentially lead to better overall family functioning, which could positively impact upon behavioral problems. In point of fact, pets provide a pivotal role in family functioning (Walsh 2009), providing an attachment source which may help bring the family members together (Cain 1983; Kurdek 2008; Grandgeorge et al. 2012) and reduce stress and promote interaction between members of the family group (Allen and Blascovich 1996).

Given the suggestion that home-based interventions which focus on parent-child interactions are worthy of further investigations (Howlin 2010), and the increasing interest in the role of animal-assisted therapy (AAT) in the treatment of mental health disorders (e.g., Barker and Wolen 2008; O'Haire 2010), our aim was to explore the role of pet dogs in the homes of children with autism. Specifically, we aimed to investigate the potential of dogs to improve family functioning in families with children with autism and explore the effects of pet dogs on anxiety in these children.

\section{Methods}

Testing procedures were carried out in accordance with the ethical standards of the University of Lincoln Ethical Committee and with the Helsinki Declaration of 1975, as revised in 2000.

\section{Recruitment}

Participants were recruited to take part in the study if their child had a confirmed diagnosis of ASD. The diagnosis was confirmed verbally by the parents. All children had received a clinical diagnosis through Children and Adolescent Mental Health Services (CAMHS) in accordance with the DSM-IV criteria (APA 2013).

Parents of children diagnosed with ASD looking to acquire a pet dog were recruited on a voluntary basis via Dogs for the Disabled's PAWS (Parents Autism Workshops and Support) network (http://paws.dogsforthedisabled.org). The PAWS program involves a series of three workshops that educate parents about dog behavior, welfare, and training, whilst advising on the suitability and integration of pet dogs into families with children with ASD. In addition, postings on websites 
Pet Dogs Improve Family Functioning and Reduce Anxiety in Children with Autism Spectrum Disorder

and social networks related to Dogs for the Disabled and the National Autistic Society (NAS), and word of mouth were used to increase the number of participants. Demographic data relating to the child, dog, and family were collected. A control group of parents who did not acquire dogs were recruited (delayed by 8 months to ensure the volume of data could be collected at the specified inter-sample timescale) through local networks and word of mouth in addition to the above routes, and sampled at matched times. The times at which data were collected were: (i) Baseline (BL); up to 17 weeks before acquiring a dog in the intervention group, (ii) Post-intervention (PI); 3-10 weeks post-dog acquisition; (iii) Follow-up (FU); 25-40 weeks post-dog acquisition. Although the terms post-intervention and follow-up are typically used to describe measurement after the completion of an intervention, the unique nature of dog ownership means that dogs live within the families for an indefinite period. Therefore, we refer to these terms in relation to the start of the intervention (acquiring a dog), rather than completing the intervention (e.g., the death of the dog or it being rehomed). These terms are used in the interest of continuity with our previous reports of data collected during this intervention (Wright et al. 2015).

\section{Participants}

Intervention Group: Ninety-three parents were recruited for the intervention group; of these, 82 completed the baseline sample. Eight of the 11 who dropped out before the baseline sample reported that they decided not to get a dog within the timescale of the study (5 of these transferred to the control group), two acquired a dog prior to baseline and so were excluded; the remaining parent was uncontactable.

Sixty parents completed both the $\mathrm{BL}$ and PI samples; 22 dropped out between $\mathrm{BL}$ and $\mathrm{Pl}$. Eleven of these reported that they would not be acquiring a dog within the study timescale ( 1 of these transferred to the control group), two were outside of the PI sampling window, two requested to drop out of the research, four were uncontactable, one got a trained assistance dog, two acquired dogs but subsequently rehomed them prior to the PI sample (reasons: one parent reported that the dog was biting the children, one parent was allergic to the dog).

Forty-two parents completed all three sample points (baseline, post-intervention, and follow-up); 18 dropped out between PI and FU, and these eight rehomed the dogs (6 due to child-dog issues; one due to child problems unrelated to the dog; one due to dog training problems unrelated to the child), one requested to drop out of the research, one was uncontactable, and seven were outside of the study timescale (i.e., date of acquisition was too late for follow-ups to be completed).

Control Group: Thirty-two parents were recruited for the control group and of these 28 completed all three samples. Three of the four who dropped out acquired a dog (one before baseline who transferred to the intervention group; one before post-intervention; and one before follow-up). The fourth drop-out completed two interviews but was not contactable for the follow-up. Regarding the intervention group, 95.2\% $(n=40)$ were recruited via the PAWS network and $4.76 \%(n=2)$ from other adverts. In contrast, $32.1 \%(n=9)$ of the controls were recruited from the PAWS network and $67.9 \%(n=19)$ through local autism networks. There were significantly more recruits from the PAWS network in the intervention group $\left(\chi^{2}=31.848, d f=1, p<0.001\right)$.

\section{Demographics}

Demographics for the intervention and control groups are displayed in Table 1. All children had a confirmed diagnosis of ASD. There was a significant difference in diagnoses between groups in the main population, with a higher proportion of Asperger's/High Functioning Autism and 
Table 1. Demographic characteristics of the intervention and control group.

\begin{tabular}{|c|c|c|}
\hline Demographic Item & $\begin{array}{l}\text { Intervention } \\
\text { (Total } n=42 \text { ) }\end{array}$ & $\begin{array}{c}\text { Control } \\
(\text { Total } n=28)\end{array}$ \\
\hline \multicolumn{3}{|l|}{ Gender } \\
\hline Female children & $8(19 \%)$ & 7 (25\%) \\
\hline Male children & $34(81 \%)$ & $21(75 \%)$ \\
\hline Female parents & 38 (90\%) & $26(93 \%)$ \\
\hline Male parents & $4(10 \%)$ & $2(7 \%)$ \\
\hline \multicolumn{3}{|l|}{ Family Structure } \\
\hline One parent household & $9(21 \%)$ & $8(29 \%)$ \\
\hline Two parent household & $33(79 \%)$ & $20(71 \%)$ \\
\hline No siblings & $6(14 \%)$ & $9(32 \%)$ \\
\hline One sibling & $22(52 \%)$ & $11(39 \%)$ \\
\hline Two siblings & $9(21 \%)$ & $7(25 \%)$ \\
\hline Three siblings & $3(7 \%)$ & $0(0 \%)$ \\
\hline Four siblings & $2(5 \%)$ & $1(4 \%)$ \\
\hline \multicolumn{3}{|l|}{ Autism Diagnosis } \\
\hline Autism diagnosis & $12(29 \%)$ & $9(32 \%)$ \\
\hline Asperger's/High functioning autism & $18(43 \%)$ & $4(14 \%)$ \\
\hline Autistic Spectrum Disorder & $11(26 \%)$ & $15(54 \%)$ \\
\hline Other & $1(2 \%)$ & $0(0 \%)$ \\
\hline \multicolumn{3}{|l|}{ Language Ability } \\
\hline No language ability & $2(5 \%)$ & $3(11 \%)$ \\
\hline Single words/gestures & $6(14 \%)$ & $4(14 \%)$ \\
\hline Simple phrases/sentences & $8(19 \%)$ & $5(18 \%)$ \\
\hline Full sentences & $22(52 \%)$ & $16(57 \%)$ \\
\hline \multicolumn{3}{|l|}{ Other Animals Owned } \\
\hline Owned cat(s) & 15 (36\%) & 8 (29\%) \\
\hline Owned small furry animal(s) & $13(31 \%)$ & $5(18 \%)$ \\
\hline Owned other pets & $12(29 \%)$ & $6(21 \%)$ \\
\hline
\end{tabular}

lower proportion of ASD in the intervention group $\left(\chi^{2}=8.493, d f=3, p=0.037\right)$. This was also true for the subset completing the anxiety scales $\left(\chi^{2}=11.870, d f=3, p=0.008\right)$.

There was no significant difference in carer gender between groups in the main population $\left(\chi^{2}=0.122, d f=1, p=0.727\right)$ or the subset completing the anxiety scales $\left(\chi^{2}=0.440\right.$, $d f=1, p=0.507)$. There was no significant difference in number of parents between groups in the main population $\left(x^{2}=0.466, d f=1, p=0.495\right)$ or in the subset completing the anxiety scales $\left(\chi^{2}=1.186, d f=1, p=0.170\right)$. There was no significant difference in child gender between groups in the main population $\left(\chi^{2}=0.354\right.$, $\left.d f=1, p=0.552\right)$ or in the subset completing the anxiety scales $\left(x^{2}=0.027, d f=1, p=0.868\right)$. Child age ranged from 2 to 16 years (mean $\pm S D$ : $8.67 \pm 3.34$ ). There was no significant difference in child age between groups in the main population $\left(t_{(68)}=0.305, d f=68, p=0.761\right.$ ) or in the subset completing the anxiety scales $\left(t_{(38)}=0.335, p=0.739\right)$.

There was no significant difference in reported language ability between groups in the main population $\left(\chi^{2}=0.763, d f=3, p=0.858\right)$ or in the subset completing the anxiety scales $\left(\chi^{2}=1.843, d f=3, p=0.606\right)$. There was no significant difference in number of siblings between groups in the main population $\left(\chi^{2}=5.260, d f=4, p=0.262\right)$, but there was in the subset completing the anxiety scales $\left(\chi^{2}=8.851, d f=3, p=0.031\right)$, with a higher proportion of children having one sibling and a lower proportion having more than one sibling, compared 
Pet Dogs Improve Family Functioning and Reduce Anxiety in Children with Autism Spectrum Disorder

Table 2. Time between samples.

\begin{tabular}{lccc}
\hline & $\begin{array}{c}\text { Intervention } \\
(\boldsymbol{M} \pm \mathbf{S} \boldsymbol{D})\end{array}$ & $\begin{array}{c}\text { Control } \\
(\boldsymbol{M} \pm \mathbf{S} \boldsymbol{D})\end{array}$ & Independent Samples $t$-test \\
\hline BL-PI (days) & Range 28-144 & Range 54-171 & $t=0.158$ \\
& $(76.13 \pm 34.44)$ & $(76.96 \pm 23.93)$ & $p=0.875$ \\
\hline PI-FU (days) & Range 144-245 & Range 98-208 & $t=0.405$ \\
& $(188.55 \pm 21.56)$ & $(186.21 \pm 23.20)$ & $p=0.687$ \\
\hline BL-FU (days) & Range 177-344 & Range 248-277 & $t=0.590$ \\
& $(264.66 \pm 35.75)$ & $(261.08 \pm 8.52)$ & $p=0.558$ \\
\hline
\end{tabular}

$\mathrm{BL}=$ Baseline; $\mathrm{PI}=$ Post-intervention; FU = Follow-up.

with the control group. There was no significant difference between the intervention group and control group in ownership of cats (main population: $\chi^{2}=1.82$, $d f=2, p=0.501$; anxiety sub-set: $\left.\chi^{2}=4.80, d f=2, p=0.09\right)$, small furry animals $\left(\chi^{2}=1.51, d f=1, p=0.27\right.$; anxiety sub-set: $\left.\chi^{2}=0.636, d f=1, p=0.540\right)$, or other animals $\left(\chi^{2}=1.21, d f=2, p=0.545\right.$; anxiety sub-set: $\left.\chi^{2}=0.309 d f=1, p=0.637\right)$.

In the intervention group 36 families acquired a single dog; two families acquired two dogs at the intervention time. The age of the dogs at acquisition ranged from 1.75 to 84 months (mean \pm SD: $5.31 \pm 13.19$ ). There were 19 male and 25 female dogs. Fifteen were crossbreeds and 29 were purebred, from 11 different breeds: nine Labrador Retrievers (two acquired by one family), four Golden Retrievers, three German Shepherd Dogs (two acquired by one family), three Cavalier King Charles Spaniels, two Miniature Schnauzers, two Cocker Spaniels, one Sussex Spaniel, one Jack Russell Terrier, one West Highland White Terrier, one Border Collie, one Fox Terrier, and one Bernese Mountain Dog. Most $(84.1 \%, n=37)$ were acquired from breeders; $9.1 \%(n=4)$ from rescue centres; $6.8 \%(n=3)$ from other sources.

There was no significant difference in sampling timescales between groups for the main population or subset completing the anxiety scales (Table 2).

\section{Data Collection}

The Family Assessment Measure-III (General Scale) provides a quantitative index of family strengths and weakness and is based on the Process Model of Family Functioning that integrates different approaches to family therapy and research (see Skinner, Steinhauer and Sitarenios 2000). The brief version of this scale (Brief FAM-III) was selected for this study as it is ideal for monitoring family functioning over time or during the course of treatment. The FAM-III (Brief) was completed via telephone by the parent (main carer) at the three sample points: baseline, post-intervention, and follow-up. Control group participants were sampled at matched times.

Child anxiety was measured using the widely validated parent-report measure Spence Children's Anxiety Scale (SCAS, Spence 1998) (or preschool version for younger or more severely affected children). The SCAS is comprised of seven subscales: Total Anxiety, Panic Attack/Agoraphobia, Generalized Anxiety, Social Anxiety/Phobia, Obsessive Compulsive Disorder, Physical Injury Fears, and Separation Anxiety. The pre-school version does not contain the Panic Attack/Agoraphobia subscale. The assessment of child anxiety was introduced at a later time in the study; as such, child anxiety was assessed in a sub-population totaling 40 participants. In the intervention group 14 participants completed the scale, of which three related to female children, with a mean age $( \pm S D)$ of 9.4 years $( \pm 1.4)$. In the control group 
26 participants completed the scales, of which five were completed for female children, with a mean age $( \pm S D)$ of 9.1 years $( \pm 3.9)$.

\section{Data Analysis}

Total FAM-III scores were used for data analysis. For the anxiety scales, the scores were converted to percentage of total possible score for each of the subscales ( $n$ subscales for Spence version, $n$ subscales for the preschool version), since a mixture of Spence and preschool versions were used depending upon what was considered most appropriate to the child's age and verbal ability (although completed by the parent, wording on the Spence version was not applicable to non-verbal children). This enabled scores from the two versions of the anxiety scale to be incorporated into a single dataset, preventing further reduction of the sample size.

Data were found to be normally distributed (Kolgomorov-Smirnov $p>0.05$ ). Summary statistics were calculated and plotted to facilitate visual inspection of results, prior to statistical analysis. FAM-III scores were analyzed using mixed-model ANCOVAs, with group (intervention versus control) as a between subjects factor, sample period (post-intervention and follow-up) as a within subjects factor, and baseline as a covariate (to control for artifacts related to differences in baseline measures between the two groups, which are apparent in the graphed data). Effect sizes are reported using Partial-eta squared $\left(\eta_{p}^{2}\right)$ for which $0.01=$ small effect, $0.06=$ medium effect, and 0.14 = large effect (Cohen 1988). Post-hoc tests were completed using Pairwise Comparisons with Bonferroni corrections. FAM-III scores were also investigated further in order to assess clinical relevance by exploring changes from baseline to follow-up in relation to the threshold indicating families are experiencing difficulties. Changes within groups in the proportion of caregivers scoring in the difficulty range at baseline versus follow up were assessed using McNemar's chi-squared tests.

Because anxiety data were collected on a sub-population of a relatively small data set (particularly in the case of the intervention group), with the aim of collecting data for future research in this under-explored area of human-animal interaction, we report mean and percent data for the anxiety measures. This is to help prevent mis-interpretation of the data from tests of statistical inference.

\section{Results}

\section{Family Assessment Measure -Version III (Brief)}

Between subjects analysis revealed a significant effect of Group $\left(F_{(1,67)}=9.355, p=0.003\right.$, $\eta_{p}^{2}=0.122$ ), with reduced scores (reflecting reduced family difficulties/increased family strengths, see Figure 1) in the intervention group (47.94 \pm 0.99 ; Corrected Mean \pm SEM) compared with the control group (52.70 \pm 1.21$)$. There was no significant within subjects effect of Time $\left(F_{(1,67)}=0.222, p=0.639\right)$ and no significant interaction effect of Group $\times$ Time $\left(F_{(1,67)}=0.256, p=0.614\right)$. This suggests that family functioning improved in the dog-owning group compared with the control group (non-dog owning) independent of baseline scores, and this improvement was evident across sampling times.

A significant number of carers moved from above the threshold indicating family difficulties at baseline to below at follow-up within the intervention group (McNemar's $p=0.031$ ), but not within the control group (McNemar's $p=1.0$ ).

\section{Child Anxiety}

In Table 3 we report mean, SEM, and percent change data during the intervention. We also include effect sizes (Cohen's $d$ : small $=0.2$, medium $=0.5$, large $=0.8$; Cohen 1992) and 
Pet Dogs Improve Family Functioning and Reduce Anxiety in Children with Autism Spectrum Disorder

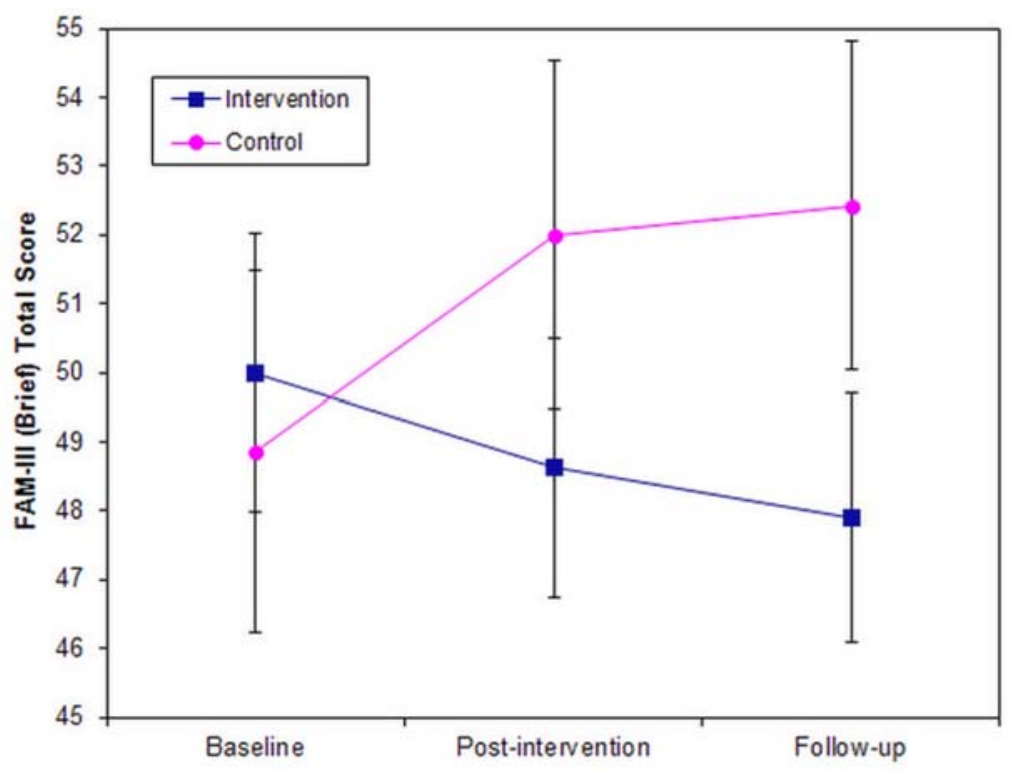

Figure 1. Family Assessment Measure III (Brief Version): Total scores for intervention group (carers acquiring a dog) and control group (carers not acquiring a dog). Error bars represent \pm 1 SEM.

power statistics. It is evident that the statistical power of the majority of the comparisons is below the generally accepted standard of $\geq 0.80$, as such we do not report inferential statistics.

Comparison of the reduction in anxiety between the intervention group and the control group revealed that Panic Attack and Agoraphobia decreased throughout the sampling times for the intervention group, with an approaching medium effect size between BL and FU. In comparison, the control group showed an initial increase in Panic Attack and Agoraphobia, followed by a small decrease. Over the sampling times (BL-FU) the intervention group showed a 24\% greater decrease in Panic Attack and Agoraphobia than the control group. Physical Injury Fears initially increased in the intervention group then decreased with a large effect size. In comparison, Physical Injury Fears in the control group decreased with a medium-large effect size over the sampling times; we discuss this point later on. Social Phobia decreased with a medium-large effect size in the intervention group over the sampling points. In the control group, despite an initial decrease, Social Phobia increased over the study period; the intervention group showed a 22\% greater decrease between BL and FU compared with the control group. Obsessive Compulsive Disorders (OCD) gradually decreased, with a medium effect size in the intervention group. In the control group small decreases were observed. There was a $26 \%$ greater reduction in OCD in the intervention group compared with the control group between BL and FU. The intervention group displayed medium-large decreases in Separation Anxiety over the study period, whereas the control group demonstrated a small decrease followed by a small increase in Separation Anxiety; between BL and FU, Separation Anxiety decreased 22\% more in the intervention group compared with the control group. Total Anxiety decreased over the study in the intervention group. In comparison, decreases in Total Anxiety were only evident at the start of the study for the control group. Total Anxiety decreased $13 \%$ more in the intervention group compared with the control group between $\mathrm{BL}$ 
Table 3. Anxiety data for the intervention and control groups.

\begin{tabular}{|c|c|c|c|c|c|c|}
\hline \multicolumn{4}{|c|}{ Mean \pm SEM } & \multicolumn{3}{|c|}{$\%$ Change } \\
\hline & BL & PI & FU & BL-PI & PI-FU & BL-FU \\
\hline \multicolumn{7}{|c|}{ Panic Attack/Agoraphobia } \\
\hline Intervention & $0.18 \pm 0.05$ & $0.18 \pm 0.04$ & $0.13 \pm 0.02$ & $\begin{array}{c}3 \%(-) \\
a=0.05, d=0.03\end{array}$ & $\begin{array}{c}28 \%(-) \\
a=0.05, d=0.03\end{array}$ & $\begin{array}{c}30 \%(-) \\
a=0.28, d=0.4\end{array}$ \\
\hline Control & $0.13 \pm 0.03$ & $0.13 \pm 0.03$ & $0.12 \pm 0.03$ & $\begin{array}{c}4 \%(+) \\
a=0.06, d=0.1\end{array}$ & $\begin{array}{c}9 \%(-) \\
a=0.07, d=0.1\end{array}$ & $\begin{array}{c}6 \%(-) \\
a=0.17, d=0.2\end{array}$ \\
\hline \multicolumn{7}{|c|}{ Physical Injury Fears } \\
\hline Intervention & $0.27 \pm 0.05$ & $0.30 \pm 0.05$ & $0.20 \pm 0.05$ & $\begin{array}{c}10 \%(+) \\
a=0.18, d=0.3\end{array}$ & $\begin{array}{c}34 \%(-) \\
a=0.98, d=1.2\end{array}$ & $\begin{array}{c}28 \%(-) \\
a=0.80, d=0.9\end{array}$ \\
\hline Control & $0.33 \pm 0.04$ & $0.26 \pm 0.03$ & $0.24 \pm 0.04$ & 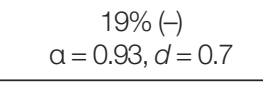 & $\begin{array}{c}8 \%(-) \\
a=0.17, d=0.2\end{array}$ & $\begin{array}{c}25 \%(-) \\
a=0.93, d=0.7\end{array}$ \\
\hline \multicolumn{7}{|c|}{ Social Phobia } \\
\hline Intervention & $0.48 \pm 0.09$ & $0.47 \pm 0.08$ & $0.36 \pm 0.06$ & $\begin{array}{c}2 \%(-) \\
a=0.05, d=0.06\end{array}$ & $\begin{array}{c}23 \%(\stackrel{)}{)} \\
a=0.54, d=0.6\end{array}$ & $\begin{array}{c}24 \%(-) \\
a=0.54, d=0.6\end{array}$ \\
\hline Control & $0.29 \pm 0.05$ & $0.26 \pm 0.05$ & $0.28 \pm 0.05$ & $\begin{array}{c}11 \%(-) \\
a=0.17, d=0.2\end{array}$ & $\begin{array}{c}10 \%(+) \\
a=0.17, d=0.2\end{array}$ & $\begin{array}{c}2 \%(-) \\
a=0.05, d=0.03\end{array}$ \\
\hline \multicolumn{7}{|l|}{$O C D$} \\
\hline Intervention & $0.30 \pm 0.06$ & $0.26 \pm 0.05$ & $0.33 \pm 0.03$ & $\begin{array}{c}14 \%(-) \\
a=0.18, d=0.3\end{array}$ & $\begin{array}{c}19 \%(-) \\
a=0.18, d=0.3\end{array}$ & $\begin{array}{c}31 \%(-) \\
a=0.41, d=0.5\end{array}$ \\
\hline Control & $0.19 \pm 0.03$ & $0.19 \pm 0.04$ & $0.18 \pm 0.05$ & $\begin{array}{c}0 \% \\
a=0.05, d=0.01\end{array}$ & $\begin{array}{c}5 \%(-) \\
a=0.07, d=0.09\end{array}$ & $\begin{array}{c}5 \%(-) \\
a=0.06, d=0.05\end{array}$ \\
\hline \multicolumn{7}{|c|}{ Generalised Anxiety Disorder } \\
\hline Intervention & $0.37 \pm 0.05$ & $0.34 \pm 0.06$ & $0.33 \pm 0.05$ & $\begin{array}{c}8 \%(-) \\
a=0.18, d=0.3\end{array}$ & $\begin{array}{c}4 \%(-) \\
a=0.06, d=0.07\end{array}$ & $\begin{array}{c}11 \%(-) \\
a=0.18, d=0.3\end{array}$ \\
\hline Control & $0.36 \pm 0.04$ & $0.29 \pm 0.04$ & $0.32 \pm 0.04$ & $\begin{array}{c}20 \%(-) \\
a=0.84, d=0.6\end{array}$ & $\begin{array}{c}12 \%(+) \\
a=0.17, d=0.2\end{array}$ & $\begin{array}{c}10 \%(-) \\
a=0.31, d=0.3\end{array}$ \\
\hline \multicolumn{7}{|c|}{ Separation Anxiety } \\
\hline Intervention & $0.49 \pm 0.07$ & $0.40 \pm 0.07$ & $0.30 \pm 0.06$ & $\begin{array}{c}9 \%(-) 26 \%(-) 32 \%(- \\
a=0.28, d=0.4\end{array}$ & $a=0.41, d=0.5$ & $a=0.68, d=0.7$ \\
\hline Control & $0.27 \pm 0.04$ & $0.26 \pm 0.05$ & $0.24 \pm 0.04$ & $\begin{array}{c}12 \%(-) \\
a=0.07, d=0.1\end{array}$ & $\begin{array}{c}12(+) \\
a=0.07, d=0.1\end{array}$ & $\begin{array}{c}10 \%(-) \\
a=0.017, d=0.2\end{array}$ \\
\hline \multicolumn{7}{|l|}{ Total } \\
\hline Intervention & $0.33 \pm 0.05$ & $0.40 \pm 0.05$ & $0.30 \pm 0.04$ & $\begin{array}{c}5 \%(-) \\
a=0.11, d=0.2\end{array}$ & $\begin{array}{c}21 \%(-) \\
a=0.68, d=0.7\end{array}$ & $\begin{array}{c}25 \%(-) \\
a=0.79, d=0.8\end{array}$ \\
\hline Control & $0.26 \pm 0.03$ & $0.23 \pm 0.03$ & $0.23 \pm 0.03$ & $\begin{array}{c}12 \%(-) \\
a=0.69, d=0.5\end{array}$ & $\begin{array}{c}0 \% 1 \\
a=0.05, d=0.01\end{array}$ & $\begin{array}{c}2 \%(-) \\
a=0.69, d=0.5\end{array}$ \\
\hline
\end{tabular}

$\mathrm{BL}$ = Baseline; $\mathrm{PI}=$ Post-Intervention; FU = Follow-Up; $(-)$ = Decrease in anxiety; $(+)$ = Increase in anxiety; $a=$ Statistical power; $d$ = Cohen's effect size.

and FU. These data show that over time anxiety reduced more in the intervention group compared with the control group, suggesting that pet dog ownership may help reduce anxiety in children with ASD. 
Pet Dogs Improve Family Functioning and Reduce Anxiety in Children with Autism Spectrum Disorder

\section{Discussion}

Two key finding emerged from our study: family functioning improved in the dog-owning (intervention) group compared with the non-dog owning (control) group, and child anxiety decreased more noticeably in the dog owning group compared with the non-dog owning group.

With regard to family functioning, those families that were in the dog-owning group demonstrated a greater increase in family strengths (reduced family difficulties) compared with the non-dog owning group, and a significant number of families moved from above the threshold (indicating family problems) to below the threshold (indicating an increase in family strengths). The increase in family strengths was not dependent upon the sampling time at which the parents were interviewed, suggesting that dog ownership brings prompt benefits to family functioning (post-intervention sampling range: 3-10 weeks) and these improvements are stable over at least short time periods (follow-up sampling range: 25-40 weeks). We are currently conducting a long-term follow-up study (3 years post-dog ownership) to determine if there are more enduring changes.

To date there are few studies that have looked at the effects of dogs on family functioning in the homes of children with ASD. One recent study highlighted how an assistance dog can promote family functioning by allowing for normal family routines and behaviors, such as shopping together as a family (Burgoyne et al. 2014). The present study supports and extends these findings, showing that a pet dog (as opposed to a trained assistance dog) can also improve family functioning, by increasing family strengths. This may be achieved through reducing stress in the family, and the potential for pet dogs to reduce parental stress has recently been demonstrated (Wright et al. 2015). A study by Allen et al. (1991) showed that stress levels reduced more in the presence of a dog compared with a friend, indicting the unique processes involved in human-animal interactions as opposed to human-human family interactions. Additionally, the dog represents a common talking point, a stress release, and a shared love which helps unite the family. Given that there were no significant differences in the number of other animals owned in the families (between the intervention and control groups), it seems likely that it is the addition of the dog that has brought about the benefits that we observed. Further research is required to establish the uniqueness of the dog species in bringing about these effects in comparison with other companion animals. However, it could be argued that owning a dog initiates a greater number of shared activities (playing fetch, dog walking, grooming) than owning a cat or other small furry animals; these shared activities may promote family bonding and therefore improve family functioning.

Our research team is exploring the dynamics of this relationship further to better understand how dogs can improve family life. The scale used here is appropriate for research purposes, particularly those studies that require repeat measures. However, given the brevity of it we can only say positive change in family functioning has occurred in the dog-owning group and not the control group, we cannot pinpoint in which relationships and with which individuals this change is most prominent.

Having established the importance of pet dogs and family functioning, this research provides a rationale for future studies to employ more in-depth, time-consuming scales to identify the specific relationships in which pet dogs may positively impact upon family functioning. For instance, studies could explore the effects of different owner-dog activities (number and type of mutual activities) and attachment levels on family functioning. To provide more objective measures of family functioning, family behaviors could be digitally recorded and blind-coded by a researcher. This would help prevent biases (from the family or the experimenter) affecting the 
results. However, the presence of a recording device is likely to change "normal" family behaviors and is unable to capture family interactions around the household and away from the home.

The exploratory nature of this project means that the potential importance of the relationship between pet dogs and child anxiety was not pertinent until after initial data collection had commenced. Hence, measures of anxiety were only collected on a small sub-sample of the original population ( $n=14$ in the intervention group). Clear patterns of reduced anxiety were observed in the intervention group. This supports previous studies that have reported the anxiety-reducing effects of service dogs in mental health patients (e.g., Lang et al. 2010; Mossello et al. 2011), and extends this to show that pet dogs can bring similar benefits.

Comparison of percent difference in anxiety scores between the two groups over the study period revealed that those children who acquired a pet dog (intervention group) showed a greater decrease in anxiety than those children who did not acquire a pet dog. These reductions were most evident in the domains of OCD, Panic Attack and Agoraphobia, Social Phobia, and Separation Anxiety. To date no scientific investigations report the effects of animal companionship on OCD. It is possible that the introduction of a pet dog helps prevent obsessive behaviors, particularly repetitive actions that are characteristic of many individuals with ASD (Turner 1999), by breaking the cycle through providing a stimulating point of interest (e.g., by playing a game with the dog). It is also possible that by stroking the dog the child engages in repetitive behaviors which are viewed as less problematic by their parent; as well, stroking can bring direct physiological benefits (e.g., reductions in physical stress responses; Allen et al. 1991). However, we did not collect data on the types of child-dog interactions, therefore it is not possible to support these speculations.

Panic Attack and Agoraphobia and Social Phobia are also common symptoms of ASD; many individuals with ASD are aware of their inability to appropriately engage in social interactions, which results in social disconnection (Attwood 2000). It could be speculated that the dog provides a vehicle of support and acts as a deflector in social situations and that this reduces the intensity of social interactions, reducing social phobia and agoraphobia stemming from the fear of meeting others. Another consideration is that children in the dog-owning group may have had greater experiences of social interactions. Previous studies have identified that dog owners are more likely to engage in social interaction (McNicholas and Collis 2000), therefore it may be that, through dog walking and family activities with the dog, the child has encountered a greater number of interactions with other people which has helped to alleviate their fears. Future investigations should control for the amount of time the child spent outside of the home environment and partaking in group activities (e.g., support groups, children's parties) with (or without) their dog, to explore whether exposure impacts upon social anxiety fears.

With regard to Separation Anxiety, the scale measures issues such as being alone, being away from parents, and something bad happening to the parents. It is possible that the children in the intervention group viewed the dog as a constant in their life, which helped to reduce these fears. For instance, whereas the parents may leave for work without any input from the child, the dog typically remains in the home or family environment (e.g., walking the dog as a family).

An interesting pattern of results was observed regarding the Physical Injury Fears subscale; fears decreased with a large effect in the control group throughout the study period, whereas in the intervention group fears initially increased before demonstrating a large decrease. This could reflect the fact that introducing a puppy into the house may bring increased risks of injury (e.g., tripping over the puppy, the puppy's toys, the puppy chewing heels/fingers), which may raise the child's anxiety about experiencing physical harm. However, the data show these fears 
Pet Dogs Improve Family Functioning and Reduce Anxiety in Children with Autism Spectrum Disorder

quickly dissipated; declining more evidently in the intervention group compared with the control group during the two final sampling points. This could reflect that owning a dog may help reduce the child's volatile behavior which decreases the risk and therefore fear of physical injury, or it could be that owning a dog helps improve safety in family outings by being a calming influence (Burrows, Adams and Spiers 2008).

The comparisons between the two groups reveal promise for the role of pet dogs in reducing anxiety in children with autism, although larger-scale studies are required. Due to the impaired language and communication skills of the children, it was not appropriate to question them about perceived benefits of dog ownership. As such, all interpretations are based on our understanding of ASD and the opinions gathered from qualitative interview data collected from parents (Wright et al. under review).

It is clear from the graphed data that scores of anxiety and family functioning were different between the two groups at baseline. In particular, the intervention group tended to be slightly worse (more family difficulties and greater child anxiety) than the control group. Whilst this reflects "real-life" variation and the highly individualized nature of autism, we chose to co-vary out the effects of baseline measures to ensure that any improvements between the two groups were not influenced by this difference. However, it is of potential interest to support organizations that those families that had decided to acquire a dog were those experiencing the most problems. It should be pointed out, too, that the dog-owning group was comprised of a larger number of children with Asperger's/high functioning autism compared with the control group. However, there were no significant differences in language abilities between the two groups. Given that anxiety is related to language skills (Hallet et al. 2013), this factor is unlikely to be the main explanation for the reduction in anxiety in the dog-owning group.

It is also worth noting that more participants were recruited from PAWS in the intervention group compared with the control group. This may have resulted in parents in the intervention group being biased to reporting positive effects, as it could be argued that these parents were more aware of the potential benefits that dog ownership can bring. However, PAWS focuses on informing owners how to train their dog and build positive relationships, rather than highlighting the benefits of dog ownership to overall family wellbeing. Therefore, it is unlikely that those recruited through PAWS were expecting to observe any more improvements in their family than those recruited through other sources. Additionally, no leading questions were asked (e.g., does your child sleep better since acquiring a dog) but instead were phrased objectively as laid out in the standardized tests (e.g., my child has trouble sleeping due to worry).

The effects reported here highlight the considerable promise of pet-dog ownership in improving the wellbeing of families living with autism. This exploratory study shows that acquiring a pet dog may be associated with improvements in family functioning and child anxiety. Future studies are needed to ascertain the magnitude and generalization of these effects. Nonetheless, this paper provides an important contribution to our understanding of a potential strategy to improve quality of life for families living with a child with autism, and represents a much-needed initial investigation into the value of pet dogs in families with children with autism.

\section{Acknowledgements}

We sincerely thank all of the parents who gave up their time to participate in the interviews. Thank you to the project advisory group whose support has been invaluable (Susan Aston, Jane Fossey, Denis Lane, Bob Michell, Jo Stevens, and the remaining seven anonymous members). We also thank the Big Lottery Fund for funding this research. 


\section{References}

Allen, K. and Blascovich, J. 1996. Anger and hostility among married couples: Pet dogs as moderators of cardiovascular reactivity to stress. Psychosomatic Medicine 58: 59-70.

Allen, K. M., Blascovich, J., Tomaka, J. and Kelsey, R. M. 1991. Presence of human friends and pet dogs as moderators of autonomic responses to stress in women. Journal of Personality and Social Psychology 61(4): 582-589.

American Psychiatric Association. 2013. Diagnostic and Statistical Manual of Mental Disorders. 5th edn. Washington, DC: Author.

Attwood, T. 2000. Strategies for improving the social integration of children with Asperger syndrome. Autism 4: 85-100.

Barker, S. B. and Wolen, A. R. 2008. The benefits of human-companion animal interaction: A review. Journal of Veterinary Medical Education 35: 487-495.

Batten, A., Corbett, C., Rosenblatt, M., Withers, L. and Yuille, R. 2006. Autism and Education: The Reality for Families Today. London: The National Autistic Society.

Berry, A., Borgi, M., Francia, N., Alleva, E. and Cirulli, F. 2013. Use of assistance and therapy dogs for children with autism spectrum disorders: A critical review of the current evidence. The Journal of Alternative and Complementary Medicine 19(2): 73-80.

Brereton, A. and Tonge, B. 2005. Pre-schoolers with Autism: A Parent Education and Skills Training Programme. London: Jessica Kingsley Publishers.

Burgoyne, L., Dowling, L., Fitzgerald, A., Connolly, M., Browne, J. P. and Perry, I. J. 2014. Parents' perspectives on the value of assistance dogs for children with autism spectrum disorder: a cross-sectional study. BMJ Open 4(6): e004786.

Burrows, K. E. Adams, C. L. and Spiers, J. 2008. Sentinels of safety: Service dogs ensure safety and enhance freedom and well-being for families with autistic children. Qualitative Health Research 18(12): 1,642-1,649.

Cain, A. 1983. A study of pets in the family system. In New Perspectives on our Lives with Companion Animals, 72-81, ed. A. Katcher and A. Beck. Philadelphia: University of Pennsylvania Press.

Carlisle, G. K. 2014. Pet dog ownership decisions for parents of children with autism spectrum disorder. Journal of Paediatric Nursing 29(2): 114-123.

Cohen J. 1988. Statistical Power Analysis for the Behavioral Sciences. New York: Routledge Academic.

Cohen, J. 1992. A power primer. Psychological Bulletin 112: 155-159.

Dunn, M. E., Burbine, T., Bowers, C. A. and Tantleff-Dunn, S. 2001. Moderators of stress in parents of children with autism. Community Mental Health Journal 37(1): 39-52.

Elsabbagh, M., Divan, G., Koh, Y. J., Kim, Y. S., Kauchali, S., Marcín, C., Motiel-Nava, C., Patel, V., Paula, C. S., Wang, C., Taghi Yasamy, M. and Fombonne, E. 2012. Global prevalence of autism and other pervasive developmental disorders. Autism Research 5: 160-179.

Fisman, S., Wolf, L., Ellison, D. and Freeman, T. 2000. A longitudinal study of siblings of children with chronic disabilities. Canadian Journal of Psychiatry 45: 369-375.

Ghaziuddin, M. 2002. Asperger syndrome: Associated psychiatric and medical conditions. Focus on Autism and Other Developmental Disabilities 17: 138-144.

Grandgeorge, M., Tordjman, S., Lazartigues, A., Lemonnier, E., Deleau, M. and Hausberger, M. 2012. Does pet arrival trigger prosocial behaviors in individuals with autism? PloS One 7(8): e41739.

Green, J., Charman, T., McConachie, H., Aldred, C., Slonims, V., Howlin, P. et al. 2010. Parent-mediated communication-focused treatment in children with autism (PACT): A randomised controlled trial. The Lancet 375(9732): 2,152-2,160.

Hallett, V., Lecavalier, L., Sukhodolsky, D. G., Cipriano, N., Aman, M. G., McCracken, J. T. et al. 2013. Exploring the manifestations of anxiety in children with autism spectrum disorders. Journal of Autism and Developmental Disorders 43: 2,341-2,352.

Howlin, P. 2010. Evaluating psychological treatments for children with autism-spectrum disorders. Advances in Psychiatric Treatment 16: 133-140.

Khouzam, H. R., El-Gabalawi, F. and Priest, F. 2004. Asperger's disorder: A review of its diagnosis and treatment. Comprehensive Psychiatry 45: 183-191.

Knapp, M., Romeo, R. and Beecham, J. 2007. The Economic Consequences of Autism in the UK. London: Foundation for People with Learning Disabilities.

Kurdek, L. A. 2008. Pet dogs as attachment figures. Journal of Social and Personal Relationships 25: 247-266. 
Pet Dogs Improve Family Functioning and Reduce Anxiety in Children with Autism Spectrum Disorder

Lang, U. E., Jansen, J. B., Wertenauer, F., Gallinat, J. and Rapp, M. A. 2010. Reduced anxiety during dog assisted interviews in acute schizophrenic patients. European Journal of Integrative Medicine 2: 123-127.

Lee, L., Harrington, R. A., Louie, B. B. and Newschaffer, C. J. 2007. Children with Autism: Quality of Life and Parental Concerns. London: Routledge.

Mayes, S. D., Calhoun, S. L., Murray, M. J., Ahuja, M. and Smith, L. A. 2011. Anxiety, depression, and irritability in children with autism relative to other neuropsychiatric disorders and typical development. Research in Autism Spectrum Disorders 5: 474-485.

McNicholas, J. and Collis, G. M. 2000. Dogs as catalysts for social interactions: Robustness of the effect. British Journal of Psychology 91(1): 61-70.

Mossello, E., Ridolfi, A. Mello, A. M., Lorenzini, G., Mugnai, F., Piccini, C. and Marchionni, N. 2011. Animalassisted activity and emotional status of patients with Alzheimer's disease in day care. International Psychogeriatrics 23(6): 899-905.

O'Haire, M. 2010. Companion animals and human health: Benefits, challenges, and the road ahead. Journal of Veterinary Behavior: Clinical Applications and Research 5: 226-234.

O'Haire, M. E. 2013. Animal-assisted intervention for autism spectrum disorder: A systematic literature review. Journal of Autism and Developmental Disorders 43: 1,606-1,622.

Quintero, N. 2010. Sibling adjustment and maternal well-being: An examination of families with and without a child with an autism spectrum disorder. Focus on Autism and Other Developmental Disabilities 25: 37-46.

Rosenblatt, M. 2008. I Exist: The Message from Adults with Autism in England. London: The National Autistic Society.

Sikora, D., Moran, E., Orlich, F., Hall, T. A., Kovacs, E. A., Delahaye, J., Clemons, T. E. and Kuhlthau, K. 2013. The relationship between family functioning and behavior problems in children with autism spectrum disorders. Research in Autism Spectrum Disorders 7(2): 307-315.

Silva, K., Correia, R., Lima, M., Magalhães, A. and de Sousa, L. 2011. Can dogs prime autistic children for therapy? Evidence from a single case study. The Journal of Alternative and Complementary Medicine 17(7): 655-659.

Skinner, H., Steinhauer, P., and Sitarenios, G. 2000. Family Assessment Measure (FAM) and process model of family functioning. Journal of Family Therapy 22(2): 190-210.

Smyth, C. and Slevin, E. 2010. Experiences of family life with an autism assistance dog: Placing specially trained dogs in families that have a child with autism can bring many benefits. Claire Smyth and Eamonn Slevin evaluated parents' views from a study in Ireland. Learning Disability Practice 13(4): 12-17.

Solomon, O. 2010. What a dog can do: Children with autism and therapy dogs in social interaction. Ethos 38 : 143-166.

Spence, S. H. 1998. A measure of anxiety symptoms among children. Behaviour Research and Therapy 36(5): 545-566.

Spiker, M. A., Lin, C. E., Van Dyke, M. and Wood, J. J. 2012. Restricted interests and anxiety in children with autism. Autism 16: 306-320.

Tantam, D. 2003. The challenge of adolescents and adults with asperger syndrome. Child and Adolescent Psychiatric Clinics of North America 12: 143-163.

Tonge, B. J., Brereton, A., Kiomall, M., Mackinnon, A., King, N. and Rinehart, N. J. 2006. Effects on parental mental health of an education and skills training program for parents of young children with autism: A randomised controlled trial. Journal of the American Academy of Child and Adolescent Psychiatry 45(5): 561-569.

Tunali, B. and Power, T. 2002. Coping by redefinition: cognitive appraisals in mothers of children with autism and children without autism. Journal of Autism and Developmental Disorders 32: 25-34.

Turner, M. 1999. Annotation: Repetitive behaviour in autism: A review of psychological research. Journal of Child Psychology and Psychiatry 40: 839-849.

Viau, R., Arsenault-Lapierre, G., Fecteau, S., Champagne, N., Walker, C. D. and Lupien, S. 2010. Effect of service dogs on salivary cortisol secretion in autistic children. Psychoneuroendocrinology 35(8): 1187-1193.

Walsh, F. 2009. Human-animal bonds II: The role of pets in family systems and family therapy. Family Process 48: 481-499.

White, S. W., Oswald, D., Ollendick, T. and Scahill, L. 2009. Anxiety in children and adolescents with autism spectrum disorders. Clinical Psychology Review 29: 216-229.

Wright, H., Hall, S., Hames, A., Hardiman, J., Mills, R. and Mills, D. 2015. Acquiring a pet dog significantly reduces stress of primary carers for children with Autism Spectrum Disorder: A prospective case control study. Journal of Autism and Developmental Disorders 45: 2,531-2,540. 